Voix et Images

volxetimages

\title{
Sur Neige noire. L’Oeuvre ouverte de Hubert Aquin
}

\section{Jacques Pelletier}

Volume 1, numéro 1, septembre 1975

Hubert Aquin

URI : https://id.erudit.org/iderudit/013982ar

DOI : https://doi.org/10.7202/013982ar

Aller au sommaire du numéro

\section{Éditeur(s)}

Les Presses de l'Université du Québec

\section{ISSN}

0318-9201 (imprimé)

1705-933X (numérique)

Découvrir la revue

\section{Citer cet article}

Pelletier, J. (1975). Sur Neige noire. L’Oeuvre ouverte de Hubert Aquin. Voix et Images, 1(1), 19-25. https://doi.org/10.7202/013982ar d'utilisation que vous pouvez consulter en ligne.

https://apropos.erudit.org/fr/usagers/politique-dutilisation/ 


\section{Sur Neige noire \\ L'OEuvre ouverte de Hubert Aquin}

Après un trop long silence de près de cinq ans, Hubert Aquin poursuit, avec Neige noire ${ }^{1}$, une œuvre singulière, inclassable, irréductible à tout courant majeur de notre littérature, voire même de la littérature occidentale prise globalement. Sa production ne relève en effet ni du romanesque traditionnel, ni du nouveau romanesque dont la voie a été déblayée par Butor, Robbe-Grillet et défrichée par la suite par leurs nombreux épigones, aussi bien étrangers que locaux. Bien entendu, l'écriture consciente, lucide, hyper-critique d'Aquin est plus près du nouveau roman que du roman traditionnel, mais elle n'en est pas vraiment une incarnation, et cela sans doute pour la bonne raison qu'elle trouve ses fondements dans une problématique radicalement différente. Celle des nouveaux romanciers est d'abord et avant tout littéraire: il s'agit essentiellement pour eux de revitaliser le roman, de le moderniser; c'est pour atteindre cette fin qu'ils remettent en question les conceptions traditionnelles du-personnage et de l'intrigue, qu'ils substituent au personnage plein de naguère un être fuyant, énigmatique, insaisissable et à l'intrigue linéaire et unidimensionnelle de leurs prédécesseurs une intrigue morcelée, fragmentée, pleine de télescopages temporels, foncièrement ambiguë et déroutante. Mais cette révolution importante, ce renversement majeur répond en premier lieu à un impératif littéraire et la fonction de l'écriture n'est pas fondamentalement remise en cause: la création d'un roman nouveau est justifiée par le besoin de trouver une forme pouvant le plus justement rendre compte de la réalité actuelle, mais la nécessité même de l'écriture romanesque, sa raison d'être, sa signification n'est pas interrogée, mise en procès.

Chez Aquin, au contraire, cette question est primordiale et constitue l'arrière-plan, le champ d'intelligibilité de ses deux premières productions romanesques. Patricia Smart ${ }^{2}$ a bien montré que la dialectique fondamentale de ses deux premiers récits était l'opposition de l'art et de la réalité historique : l'art a-t-il sa raison d'être dans une société coloniale où l'action, pour un révolutionnaire, doit d'abord être concrète, pratique, politique? Dans quelle mesure la pratique artistique ne constitue-t-elle pas un dérivatif, un obstacle à l'action, et par conséquent une activité parasitaire, inessentielle, nuisible à la tâche prioritaire qu'est l'œurre de décolonisa- 
tion? Cette problématique fournit à la fois la thématique majeure des deux premiers romans d'Aquin et leur principe de composition. On voit qu'elle est très différente de celle des nouveaux romanciers, et si les textes d'Aquin présentent malgré tout un certain air de parenté avec ceux de ces auteurs, on verra que c'est parce que, comme eux, Aquin considère d'abord ses écrits comme des fictions et non comme des décalques, reproductions plus ou moins abâtardies de quelque réalité externe qu'ils viseraient à mettre en forme. C'est à ce problème de structure que je m'attarderai donc exclusivement dans le bref texte que voici; je me propose de montrer comment il a été abordé et résolu par Aquin jusqu'à Neige noire et dans ce dernier roman qui apparaît, tout à la fois, comme un approfondissement et une rupture avec les œuvres antérieures.

La structure de Prochain Épisode, on s'en souviendra, est somme toute assez simple: le récit est pris en charge par un narrateur immobilisé dans une clinique psychiatrique qui désire tout à la fois gagner du temps et donner une signification à la période creuse de sa réclusion en écrivant un roman de type policier-politique, projet qu'il ne réussira pas à réaliser pour la bonne raison qu'il est inincarnable par définition, le seul «roman » possible étant alors pour Aquin celui de l'action directe révolutionnaire. celui qui s'écrit dans le sang avec des rafales de mitraillettes. Il s'agit d'un récit au fond relativement transparent, ne comportant qu'un seul véritable dédoublement et un nombre restreint de trompe-l'œil et de fausses pistes.

Les choses vont vraiment commencer à se compliquer, pour ainsi dire, dans Trou de mémoire. On assiste dans ce second roman à une multiplication des instances narratives. Le récit est d'abord pris en charge par un narrateur périphérique, Ghezzo-Quénum, un personnage exotique qui, de sa brousse africaine, écrit une lettre au héros principal, P. X. Magnant, un pharmacien dont on nous donnera ensuite à lire le roman qu'il a entrepris d'écrire sur son passé récent. Ce texte fait lui-même l'objet de commentaires, de «notes" de deux autres narrateurs: un énigmatique éditeur et un non moins mystérieux personnage qui se contente de faire suivre ses réflexions de ses seules initiales, R. R. Ces deux narrateurs monopolisent la parole dans des proportions inégales, le texte de l'éditeur étant beaucoup plus long que celui de R. R. Soulignons encore qu'une cinquième instance narrative est mise à contribution dans la personne d'un certain Mullahy qui fait une brève apparition dans les dernières pages du roman. Ainsi présentées, les choses paraissent relativement simples. Cependant il n'en est rien dans le roman où les divers narrateurs commentent les textes des uns et des autres. Si bien qu'il est extrêmement difficile de repérer le véritable meneur de jeu du récit. Difficulté première que vient aggraver le statut indifférencié des narrateurs qui, à l'exception de $R$. $R$., ne constituent peut-être qu'un seul et même personnage ${ }^{3}$. Machinerie plus complexe que Prochain Épisode, Trou de mémoire lui est cependant apparenté par sa thématique nationaliste, sa structure para-policière ${ }^{4}$ et l'esthétique de "la fureur et de l'incantation " qui le caractérisent.

Avec l'Antiphonaire, s'ouvre une nouvelle période dans l'œuvre d'Aquin. La question nationale québécoise qui constitue la thématique 
principale sinon unique des premiers romans disparaît à toutes fins pratiques dans ce troisième roman et dans Neige noire. Tout au moins en tant que thématique explicite, ce qui n'empêche pas bien entendu de lire et d'interpréter ces récits comme des expressions d'une société coloniale reproduisant, au niveau formel, la condition d'aliénation des Québécois. Principe d'organisation, de mise en forme des premiers romans, la question nationale sera relayée désormais par une nouvelle problématique, dont le thème central est la recherche de valeurs authentiques, du sens à donner à l'existence. Ce qui aura pour conséquence l'apparition de nouvelles structures romanesques. Dans l'Antiphonaire on trouve une prodigieuse réalisation de ce que Gide appelait une "mise en abîme", le récit se situant à la fois ici et maintenant, c'est-à-dire en Californie et à Montréal à l'époque contemporaine, et dans le passé, à l'époque de la Renaissance. Le roman est conduit à deux niveaux qui se chevauchent continuellement de part en part du texte. La partie du roman "en abîme" apparaît dans une certaine mesure autonome, indépendante du récit premier dans lequel elle est enchâssée, possédant son intérêt propre. En tant qu'entité distincte, elle est d'ailleurs de facture assez traditionnelle; elle est cependant dynamisée et modernisée par les considérations du narrateur, Christine Forestier, héroïne du récit premier qui se projette et se reconnaît dans différents personnages du récit second: Renata Belmissieri, Jean-Étienne de Calcar, son "double masculin », Antonella Zimara. Ce phénomène de dédoublement se produit aussi à l'intérieur même du récit second, le théoricien Beausang se prolongeant dans les personnes des abbés Chigi et Zimara. Par cette dimension l'Antiphonaire se rattache de près, malgré ses innovations, aux romans précédents; on a toujours affaire à un texte où prolifèrent les métamorphoses et les jeux de miroirs et que surcharge délibérément tout un appareil ornemental formé ici pour l'essentiel d'une débauche d'érudition sur la dense et riche période de la Renaissance: la nomenclature pharmaceutique a fait place à l'érudition historique, mais la fonction de ces insertions n'a pas changé; il s'agit de surprendre le lecteur, de l'épater, de lui donner le vertige et ce faisant, de lui montrer concrètement que le roman est d'abord et avant tout une fiction, un texte: cet étalage de connaissances a d'abord une valeur ironique. On voit que ce troisième roman à la fois prolonge et dépasse dans une structure nouvelle les romans précédents. Cette rupture va cependant apparaître de manière beaucoup plus concluante et décisive dans Neige noire.

Au niveau même de sa présentation matérielle, le livre s'inscrit sous le signe de l'ambiguïté. Une première série d'oppositions apparaît en effet dans la présentation graphique du titre du roman, le mot neige étant imprimé en noir et le mot noire étant, lui, imprimé en rouge. Déjà surgit une double inversion: la neige, associée généralement à la blancheur, l'est ici à la noirceur, et cette noirceur est elle-même métamorphosée en rougeur. Au dos du livre, même ambiguïté: la figure de l'auteur qu'on nous donne à voir est celle tirée d'un négatif de photographie, le représentant comme masqué. A côté de cette photo truquée, on trouve un texte tiré du roman, faisant l'apologie du masque en tant qu'instrument de libération de l'indi- 
vidu qui, sous sa protection, peut se livrer sans crainte à ses émotions les plus profondes et les plus viscérales, texte qui se termine par la phrase sybilline suivante: "L'idéal, même, serait d'imaginer le lecteur d'un livre portant un masque dont il pourrait même se servir comme signet. "Enfin, signalons encore à ce stade des approches du livre, l'épigraphe ambivalente empruntée au philosophe tourmenté de l'existence, Soren Kierkegaard: « Je dois maintenant à la fois être et ne pas être."

L'originalité qui se manifeste à ce niveau n'offre pourtant qu'un avantgoût de ce que le roman contient: une utilisation spectaculaire et révolutionnaire du langage cinématographique à des fins purement romanesques. Expérience qu'à ma connaissance Aquin est le premier à réaliser d'une manière aussi conséquente et avec autant de bonheur.

Bien entendu, la question des rapports du cinéma et du roman n'est pas nouvelle. Depuis longtemps le cinéma fascine les romanciers et on sait que les grands écrivains américains de l'entre-deux-guerres lui ont emprunté un certain nombre de techniques. Claude Edmonde Magny l'a bien montré dans la première partie de son livre consacré à l'Âge du roman américain. Et dans la période plus immédiatement contemporaine, un Robbe-Grillet, en France, a manifestement été influencé par les procédés d'écriture du cinéma - au point de devenir cinéaste! - : cela apparaît très clairement dans sa production romanesque.

Les points communs entre les deux genres ne manquent pas. Le cinéma et le roman sont tous deux des instruments de représentation du réel, des langages, des récits dont l'un, le roman, emploie exclusivement des mots, tandis que l'autre, le cinéma, utilise des mots bien sûr mais d'abord et avant tout des images et des sons. Et dans les deux cas, un certain nombre de problèmes similaires se posent. Celui d'abord du cadrage, c'est-à-dire du découpage de l'image, du fragment de réalité à isoler et à reproduire dans le texte ou à l'écran. Celui ensuite de la détermination de l'angle de prise de vue, c'est-à-dire du choix du lieu où sera localisé le narrateur qui décrit dans le cas du roman, le caméraman dans le cas du cinéma. Celui encore de la délimitation du mode de vision (objective, subjective, expressive, etc.). Celui enfin de la composition et du montage (linéaires, inversés, alternés et parallèles). Le romancier et le cinéaste ont à faire face à ces différents problèmes et, en ce sens, on peut affirmer que leur travail n'est pas aussi dissemblable qu'on le croit généralement.

Ceci dit, le roman d'Aquin est profondément original en ce qu'il met à contribution pour des fins romanesques le langage cinématographique que l'auteur, cinéaste, connaît à la perfection et manie en virtuose. Le récit se présente en effet sous l'aspect d'un scénario de cinéma, texte premier qui est lui-même l'objet de commentaires de la part d'un narrateur second (en «voix off»), commentaires par lesquels le romanesque est vraiment injecté dans un texte qui, tel quel, aurait pu effectivement n'être qu'un scénario. C'est dans ces commentaires que figurent notamment les nombreuses réflexions sur le temps qui constituent le noyau central de la thématique du roman. J'y reviendrai.

“Le film, dit quelque part Eva Vos, héroïne principale de la deuxième 
moitié du récit, raconte l'histoire d'un comédien qui quitte son métier pour faire un film qui ne sera que le compte-rendu de sa vie à partir de ce moment-là. C'est comme un film dans un film. " Ce texte, qui donne une des clefs du récit, notons-le, est lui-même dans le scénario du film en cours d'élaboration... L' «histoire" de Neige noire est en effet celle d'un comédien, Nicolas, qui abandonne son métier d'acteur pour écrire un scénario dans lequel lui-même et sa maîtresse Sylvie seront les protagonistes principaux. Le début du récit est centré sur les problèmes préliminaires qui se posent à Nicolas, devenu scénariste, puis sur les rapports beaucoup plus sérieux, cruciaux du réel et de la fiction. Car le film n'est rien d'autre qu'une sorte d'autobiographie de Nicolas à partir du moment où il a décidé de le réaliser. Si bien que le film avancera dans la mesure où dans sa vie des choses se produiront. Ainsi par exemple Sylvie doit mourir dans la réalité avant de pouvoir entrer dans la fiction. Le cinéma, ainsi conçu et pratiqué, est un exercice dangereux et lorsque Eva, qui a succédé à Sylvie dans la vie amoureuse de Nicolas, le comprend, elle ne veut plus jouer le jeu. Elle quitte Nicolas et se réfugie chez Linda Noble, autre double de Sylvie, avec qui elle partage un amour profond à forte saveur mystique. Notons que cette "conclusion" figure aussi dans le scénario de Nicolas, ce qui ne va pas sans laisser songeur...

Le scénario par ailleurs raconte l' "histoire» des difficultés de Nicolas pour réaliser son film et ses liaisons amoureuses. A ce niveau, le texte est relativement transparent: Nicolas, un jeune comédien montréalais, se propose à vingt-huit ans de refaire sa vie, de changer de métier et d'approfondir son amour pour Sylvie Dubuque, sa maîtresse. C'est à cette fin que les deux amants s'envolent pour la Norvège où effectivement leur amour s'épanouira, Nicolas retrouvant notamment «quelque chose qui ressemble au goût de vivre". (p. 60) Leur passion va atteindre son point culminant Iorsqu'ils approcheront du Pôle, point extrême symbolisant un absolu peutêtre inaccessible. Car cette osmose profonde, cette communion que connaîtront Eva et Linda Noble, Nicolas et Sylvie ne réussiront pas à l'atteindre, leur amour étant miné de l'intérieur par la présence d'une ombre menaçante, celle de Michel Lewandowsky, ancien amant de Sylvie dont Nicolas n'arrive pas à se débarrasser. Or nous saurons plus tard que ce Michel Lewandowsky est le père de Sylvie Dubuque, de son vrai nom Lewandowsky. Et la liaison incestueuse du père et de la fille sera elle-même dédoublée plus avant dans le récit par Lewandowsky et Eva Vos. Ici encore, comme dans les romans précédents, on retrouve les procédés caractéristiques de la manière d'Aquin: dédoublements, mises en abîme, effets de trompel'œil, etc. ${ }^{5}$.

Ce scénario est lui-même enchâssé dans un commentaire qui "explique la publication de ce.qu'il enchâsse et reproduit, structuralement, le stratagème de la pièce dans la pièce, mais à l'envers: ce n'est pas une insertion du plus petit dans le plus grand, mais ju plus grand dans le plus petit, ce qui évoque généralement une idée de resserrement. Or le resserrement définit fort bien le procédé de l'écriture, tandis que la réalisation cinématographique implique une dilatation ". (p. 195) Ailleurs, le commen- 
tateur oppose à nouveau l'écriture au cinéma, en tant qu'arts respectivement de l'immobilité et du mouvement. Or en recourant au langage cinématographique, Aquin compense dans une large mesure les effets pétrifiants de l'écriture. D'une part il peut procéder à des raccourcis, des ellipses totales, en faisant l'économie des fameux «résumés sommaires» dont arrive difficilement à se passer le roman. Et par ailleurs il peut rapprocher dans un même espace narratif des événements qui, dans la réalité évoquée par le texte, se déroulent en des lieux et en des moments très différents. L'esthétique de la «superposition " ne sous-entend donc pas seulement le film, comme l'affirme le commentateur à la page 227, mais le texte qu'on nous donne à lire qui n'est pas un véritable scénario, mais un roman empruntant le visage d'un scénario: "Celui qui s'est rendu jusqu'ici sait que ce n'est pas un film qu'il regarde par les yeux d'un spectateur, mais un livre qu'il continue lui-même de feuilleter en tremblant. » (p. 253)

II ne faut pas oublier par surcroît que le commentaire est indissociable du scénario, que ces deux fragments narratifs forment ensemble le roman dont le thème central est sans doute le temps. La méditation sur le temps apparaît surtout dans les textes constituant le commentaire du scénario. Elle se présente d'abord sous la forme d'une réflexion sur l'opposition continuité/discontinuité. La continuité, "enchaînement sans ellipses, sans bonds, sans hoquets, sans coupures, sans excursus latéraux " n'est en réalité qu'une notion construite, qu'une fiction construite après coup «par un effet de perspective». En fait, il n'existe rien de tel, “ le cours de la vie est chaotique et imprévisible» (p. 48), “le flux temporel est un torrent impur qui transporte, dans sa violence fluide, l'imprévisible existence qui se fait au fur et à mesure que l'eau matricielle descend vers une vallée, vers n'importe quelle vallée" " (p. 49) De cette analyse, le commentateur tire une conclusion applicable au scénario, "la discontinuité du film " n'étant "que le versant formel de celle de tout ce qui vit ". (p. 40) Par ailleurs, le temps qui est défini comme un élément discontinu n'existe vraiment que sur le mode du passé: «le temps perçu est forcément du passé, ce qui revient à dire que le présent a un arrière-goût de souvenir et que l'avenir projeté n'est qu'un futur souvenir, donc un passé à venir!" (p. 93) Ailleurs, le commentateur écrit encore: «le temps ne peut être que rétrospectif [...] On ne feuillette pas le temps, c'est plutôt lui qui envahit tout, infiltration morbide que rien ne combat et qui transforme toute joie en sa nostalgie et tout amour en désespoir». (p. 58-59) Le temps est donc essentiellement destructeur, principe de dépravation, comme dirait Lukacs: «Le temps lui-même, fluide fluide, n'est qu'une éponge souillée par toutes les entreprises qu'il semble encourager et qu'il enterre, les unes après les autres, dans un même bain de tristesse. Le temps est une vierge enceinte. Et si c'est un fleuve, ce fleuve est un cimetière rapide qui emporte tout, même les berges qui l'étreignent, les arbres qui le bordent, les barques qu'il porte." (p. 149) Cependant, ce caractère dépradateur du temps est en partie mis en question dans le roman par la rédemption finale d'Eva et de Linda et, à un autre niveau, par le fait qu'on peut le récupérer par l'œuvre d'art: "Le temps me dévore, mais de sa bouche, je tire mes histoires, de sa sédimentation mystérieuse, je 
tire ma semence d'éternité." (p. 254) C'est sur cette résonance proustienne que se termine le récit d'Aquin, seul passage narratif d'ailleurs qui soit écrit à la première personne du singulier et qui soit de nature à nous suggérer que l'énigmatique commentateur du scénario n'est autre que l'auteur luimême.

Cette dernière propriété distingue aussi, structuralement, Neige noire des romans précédents. Ceux-ci étaient tous des expressions plus ou moins exacerbées d'un moi qui essayait de voir clair, de donner un sens au chaos de son existence. Ici, le moi a disparu presque totalement, ne se manifestant que par cette très brève apparition à la toute fin du récit. Cela est-il un indice de ce que l'œuvre d'Aquin s'oriente maintenant vers une sorte d'objectivité de même qu'elle a peu à peu délaissé la thématique nationale? Les romans qui viendront nous le diront: Aquin n'a probablement pas fini d'étonner, mais il est si imprévisible qu'on peut difficilement imaginer quelle forme prendra sa nouvelle production romanesque. Et Neige noire nous rappelle de manière somptueuse que cette cuvre est par définition déroutante, ouverte à tous les possibles et que son auteur, comme l'a bien dit André Langevin, est sûrement l'écrivain de langue française le plus intelligent de notre époque. II est grand temps qu'on s'en rende compte, ici comme ailleurs.

Jacques Pelletier

1. Montréal, La Presse, 1974.

2. Patricia Smart, Hubert Aquin, agent double, Montréal, PUM, “Lignes québécoises ", 1973, $139 \mathrm{p}$.

3. Sur cette question, lire l'article de Jean-Pierre Martel, "Trou de mémoire: ceuvre baroque", dans Voix et images du pays VIII, Montréal, PUQ, 1974, p. 67104.

4. "Tous les romans sont policiers, c'est l'évidence même et je n'y peux rien ", écrit l' 'éditeur" de Trou de mémoire, Montréal, Le Cercle du livre de France, 1968, p. 82.

5. On trouve aussi dans le récit des motifs jouant le rôle de générateurs, pour reprendre la suggestive expression de Jean Ricardou. Le plus important est sûrement l'évocation de la ville de Natchez-Under-the-Hill. La première référence à Natchez apparaît dans une conversation entre Nicolas et Sylvie. Celle-ci demande à Nicolas de partir et de se rendre immédiatement à la dite ville, ce que Nicolas refuse. Natchez est ensuite évoqué dans une conversation entre Nicolas et Eva, dans une gravure accrochée au-dessus de la tête du lit de la chambre où se rencontrent Michel Lewandowsky et Sylvie, enfin toujours dans cette chambre au moment où l'on apprend que Lewandowsky est le père de Sylvie. A la suite de quoi, le commentateur écrit, nous facilitant ainsi la tâche d'explication: «soudain il [le spectateur] comprend que Michel Lewandowsky, celui qui a été l'amant de Sylvie, était aussi son père et qu'il n'y a donc, au terme de cette énigme, qu'une seule et même Sylvie et que le seul lien qui, depuis le début du film, permettait d'établir cette équation tragique était cette gravure de Natchez-Under-the-Hill et non pas le patronyme de celle qui est ensevelie dans le ravin du Spitzbergen $\%$. (p. 215) On pourrait effectuer une analyse du même type à propos du pendentif de Sylvie et de quelques autres objets figurant dans le roman. 